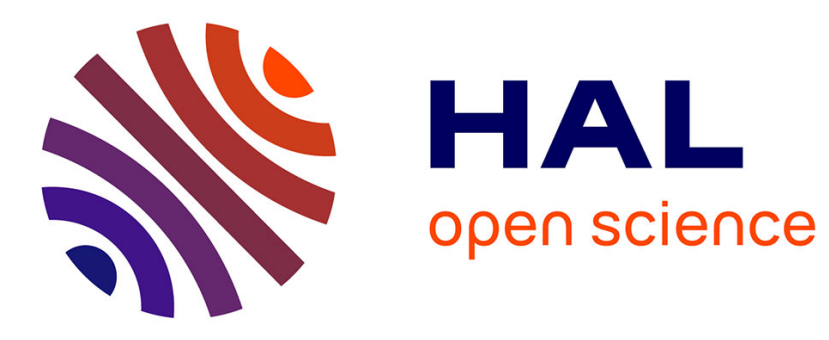

\title{
The rotational spectrum of Methylarsine
}

R.A. Motiyenko, V.V. Ilyushin, J. Demaison, N. Vogt, L. Margulès, H.

Møllendal, Jean-Claude Guillemin

\section{To cite this version:}

R.A. Motiyenko, V.V. Ilyushin, J. Demaison, N. Vogt, L. Margulès, et al.. The rotational spectrum of Methylarsine. Journal of Molecular Structure, 2020, 1213, pp.128037. 10.1016/j.molstruc.2020.128037 . hal-02569942

HAL Id: hal-02569942

https://hal-univ-rennes1.archives-ouvertes.fr/hal-02569942

Submitted on 12 May 2020

HAL is a multi-disciplinary open access archive for the deposit and dissemination of scientific research documents, whether they are published or not. The documents may come from teaching and research institutions in France or abroad, or from public or private research centers.
L'archive ouverte pluridisciplinaire HAL, est destinée au dépôt et à la diffusion de documents scientifiques de niveau recherche, publiés ou non, émanant des établissements d'enseignement et de recherche français ou étrangers, des laboratoires publics ou privés. 
Graphical abstract:
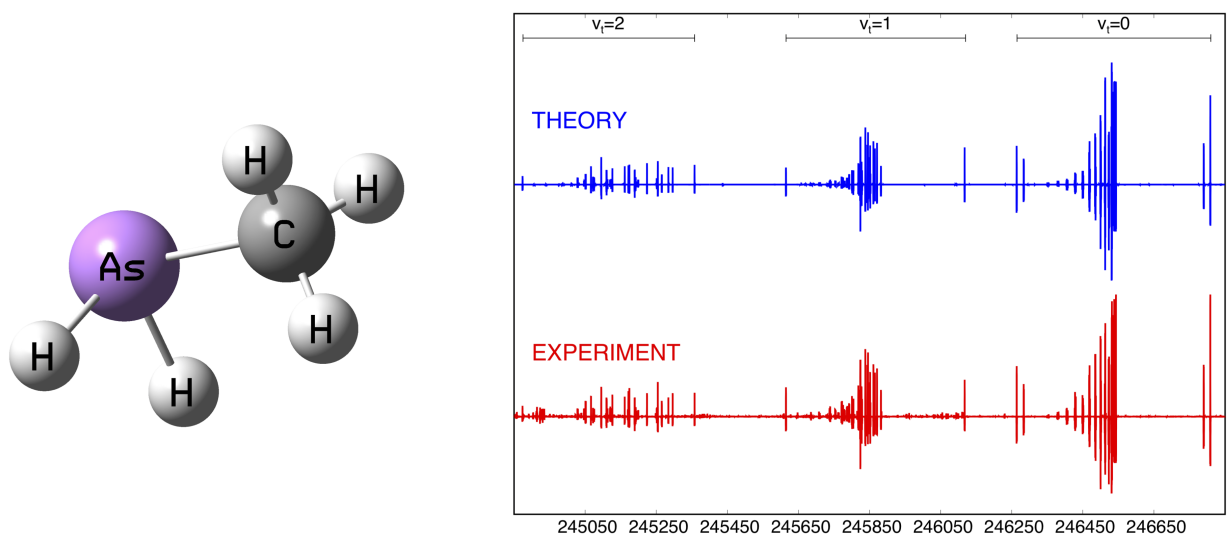

Frequency $(\mathrm{MHz})$

Highlights:

- Accurate quantum chemical calculations of the equilibrium structure of $\mathrm{CH}_{3} \mathrm{AsH}_{2}$

- A case study of a molecule with torsional LAM and relatively strong quadrupole coupling

- Analysis of the ground, first, and second excited torsional states of $\mathrm{CH}_{3} \mathrm{AsH}_{2}$ 
R. A. Motiyenko: Formal analysis, Investigation V. V. Ilyushin : Formal analysis, Validation J. Demaison : Conceptualization, Formal analysis N. Vogt: Formal analysis, L. Margulès: Investigation, H. Møllendal: Investigation, J.-C. Guillemin : Conceptualization, Investigation 


\title{
The Rotational Spectrum of Methylarsine ${ }^{\text {tr }}$
}

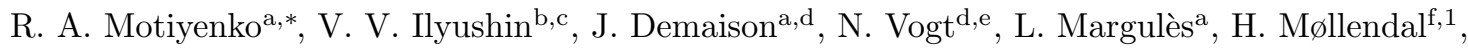 \\ J.-C. Guilleming \\ ${ }^{a}$ Université de Lille, FST, Laboratoire de Physique des Lasers, Atomes et Molécules, CNRS UMR 8523, F-59650 Villeneuve \\ d'Ascq, France \\ ${ }^{b}$ Institute of Radio Astronomy of the National Academy of Sciences of Ukraine (IRA NASU), 4, Mystetstv St., Kharkiv, \\ 61002, Ukraine \\ ${ }^{c}$ Quantum Radiophysics Department, V.N. Karazin Kharkiv National University, Svobody Square 4, 61022, Kharkiv, Ukraine \\ ${ }^{d}$ Section of Chemical Information Systems, University of Ulm, Ulm, 89069, Germany \\ ${ }^{e}$ Department of Chemistry, Lomonosov Moscow State University, Moscow, 119992, Russian Federation \\ ${ }^{f}$ Centre for Theoretical and Computational Chemistry (CTCC), Department of Chemistry, University of Oslo, P.O. Box \\ 1033, Blindern, N-0315 Oslo, Norway \\ ${ }^{g}$ Univ Rennes, Ecole Nationale Supérieure de Chimie de Rennes, CNRS, ISCR - UMR 6226, F-35000 Rennes, France
}

\begin{abstract}
The rotational spectrum of an organoarsenic compound, methylarsine, $\mathrm{CH}_{3} \mathrm{AsH}_{2}$, was studied in the frequency range 10 - $80 \mathrm{GHz}$ using the Stark-modulated spectrometer in Oslo, and in the frequency range 150 - $660 \mathrm{GHz}$ using the Lille spectrometer. The experimental work was augmented by high-level ab initio calculations. The analysis of large amplitude torsional motion in $\mathrm{CH}_{3} \mathrm{AsH}_{2}$ was performed using the rho axis method and the RAM36 code modified to take into account the nuclear quadrupole hyperfine structure. The final fit used 48 parameters (including 3 parameters of quadrupole coupling) to give an overall unitless root-mean-square deviation of 0.92 for the dataset consisting of 2753, 991, and 414 transitions belonging, respectively, to the ground, first, and second excited torsional state. Compact grouping of rotational transitions due to the small asymmetry $(\kappa=-0.9986)$, torsional splittings due to internal rotation of the $\mathrm{CH}_{3}$ group, and relatively large hyperfine splittings due to a rather large value of the arsenic nuclear quadrupole moment $\left(\chi_{a a}=35.4 \mathrm{MHz}, \chi_{c c}=-117.2 \mathrm{MHz}, \chi_{a c}=115.7 \mathrm{MHz}\right.$ ) represent an interesting case of interplay of different splittings in a molecule with a rather strong coupling between internal and overall rotations $(\rho=0.413)$.
\end{abstract}

Keywords: Methylarsine, torsional large amplitude motion, microwave spectrum, quadrupole hyperfine structure

\section{Introduction}

Methylarsine, $\mathrm{CH}_{3} \mathrm{AsH}_{2}$, is an extremely poisonous gas (b.p. $2^{\circ} \mathrm{C}$ ) with an intense garlic odour. Its pyrolysis at $300^{\circ} \mathrm{C}$ generates $\mathrm{As}, \mathrm{CH}_{4}$, and $\mathrm{H}_{2}$. It is found in some lakes because of arsenic methylation by bacterial cultures and by lake sediments [1. Methylarsine was also identified as a decomposition product of 5 arsine in the presence of trimethylgallium in the studies of metal-organic chemical vapour deposition growth processes 2.

From a spectroscopic point of view, methylarsine is an interesting case for studying the methyl top internal rotation in a molecule with a relatively strong nuclear quadrupole coupling. Due to nonzero quadrupole

\footnotetext{
This manuscript is a part of the special issue "Spectroscopy and theory of isolated molecules and molecular clusters" in memory of Dr. Jon T. Hougen.

* Corresponding author

Email address: roman.motiyenko@univ-lille.fr (R. A. Motiyenko )

${ }^{1}$ Deceased on July 13, 2016
} 
moment of ${ }^{75} \mathrm{As}$, the quadrupole hyperfine splittings in methylarsine may be of the order of several tens of $10 \mathrm{MHz}$. Furthermore, this molecule is a near-symmetric top $(A=65 \mathrm{GHz}, B-C=40.4 \mathrm{MHz}, \kappa=-0.9986)$ and, for this reason, the asymmetry splittings for many low $K$ energy levels may have the same order of magnitude as the quadrupole splittings. In addition, we have torsional splittings due to hindered internal rotation of the methyl top in $\mathrm{CH}_{3} \mathrm{AsH}_{2}$. Despite a rather heavy $\mathrm{AsH}_{2}$ frame, in this molecule, the internal rotation is characterized by a rather large coupling between internal and overall rotations. The coupling is usually defined by the absolute value of the so-called $\rho$ vector. The components of the $\rho$ vector are calculated using the following expression: $\rho_{g}=\left(\lambda_{g} I_{\alpha} / I_{g}\right),(g=a, b, c)$, where $\lambda_{g}$ are the direction cosines of the internal rotation axis of the top in the principal axis system, $I_{g}$ are the principal inertia moments and $I_{\alpha}$ is the inertia moment of the methyl top. In methylarsine, the internal rotation axis of the methyl top practically coincides with the principal axis $a$

20 thus $\lambda_{a} \approx 1.0, \lambda_{c} \approx 0.0$ ( $\lambda_{b}=0.0$ by symmetry). In addition, despite the presence of a rather heavy ${ }^{75} \mathrm{As}$ atom, $I_{a}$ is relatively small since ${ }^{75} \mathrm{As}$ atom almost lies at the principal axis $a$ and contributes only slightly to the $I_{a}$ moment of inertia. The combination of these two factors gives a rather large value of $\rho=0.413$. Thus, we have an interesting case of interplay of different splittings in a molecule with a rather large coupling between internal and overall rotations.

Very few spectroscopic studies have been devoted to methylarsine. There is a dissertation submitted in August 1973 by D. M. Levine at the Pennsylvania State University which is devoted to the methylarsine microwave spectrum measured with a Stark spectrometer in the range $17-40 \mathrm{GHz}$ [3]. The rotational constants, the nuclear quadrupole coupling constants due to ${ }^{75} \mathrm{As}$ and an approximate value for the barrier height for internal rotation of the methyl group were determined. Also, the $a$-component of the dipole so moment was determined by Stark measurements, $\mu_{a}=0.84(5) \mathrm{D}$. To the best of our knowledge this work has not been published in any peer-reviewed journal. The infrared spectrum of methylarsine has been studied at low resolution by Harvey and Wilson [4] who managed to determine a force field and measure the torsional vibration frequency.

In this paper, we present the results of our study of the torsion-rotational spectrum of methylarsine.

35 The new measurements of the methylarsine spectrum were done up to $652 \mathrm{GHz}$ and transitions belonging to the ground, first, and second excited torsional states were involved in the analysis. The analysis was done using the rho-axis-method (RAM) Hamiltonian, which takes into account quadrupole coupling in the first order of perturbation treatment as realized in the RAM36hf code (see Section 3.2). Previously, the RAM36hf code was successfully applied to the analysis of the rotational spectra of N-methylformamide [5]

40 and nitromethane 6], molecules with correspondingly 3 - and 6-fold barriers to internal rotation. In these two studies, the hyperfine interaction due to the non-zero nuclear electric quadrupole moment $Q$ of the nitrogen atom was treated but it is much weaker than the corresponding electric quadrupole moment $Q$ in methylarsine. Thus, methylarsine may be considered as a more interesting benchmark molecule for testing the RAM36hf code.

\section{Experiment}

Synthesis

Caution. Methylarsine is a pyrophoric and toxic compound; all preparations and handling must be carried out in a well ventilated hood. Dichloromethylarsine (3.2 g, $20 \mathrm{mmol})$ was slowly introduced under nitrogen in a $250 \mathrm{~mL}$ flask partially immersed in a bath cooled at $-35^{\circ} \mathrm{C}$ and containing LAH $(0.76 \mathrm{~g}, 20$ $\mathrm{mmol}$ ) in tetraglyme $(50 \mathrm{~mL})$. At the end of the addition, the mixture was stirred for $15 \mathrm{~min}$ at $-35^{\circ} \mathrm{C}$ and the flask was fitted to a vacuum line equipped with two traps. The first trap was immersed in a bath cooled at $-50^{\circ} \mathrm{C}$ to remove high boiling impurities and the second one at $-196^{\circ} \mathrm{C}$ to trap methylarsine. Methylarsine was obtained in pure form in a $45 \%$ yield $(0.83 \mathrm{~g})$.

\section{Spectroscopy}

The measurements of the rotational spectrum of methylarsine in the frequency range 10 to $80 \mathrm{GHz}$ were performed using the Stark-modulated spectrometer of the University of Oslo 7]. The spectrum was 
Table 1: Structure of methylarsine (distances in $\AA$ and angles in degrees)

\begin{tabular}{|c|c|c|c|c|c|}
\hline $\begin{array}{l}\text { Method } \\
\text { Basis set }\end{array}$ & $\begin{array}{l}\text { CCSD(T)_AE } \\
\text { pwCVTZ-PP }\end{array}$ & $\begin{array}{l}\text { CCSD(T)_AE } \\
\text { pwCVQZ-PP }\end{array}$ & $\begin{array}{l}\text { MP2_AE } \\
\text { pwCVQZ-PP }\end{array}$ & $\begin{array}{l}\text { MP2_AE } \\
\text { pwCV5Z-PP }\end{array}$ & $r_{e}^{\mathrm{a}}$ \\
\hline C-As & 1.9713 & 1.9671 & 1.9548 & 1.9541 & 1.9664 \\
\hline $\mathrm{C}-\mathrm{H}_{s}$ & 1.0856 & 1.0849 & 1.0817 & 1.0814 & 1.0846 \\
\hline $\mathrm{C}-\mathrm{H}_{a}$ & 1.0880 & 1.0873 & 1.0841 & 1.0838 & 1.0870 \\
\hline As-H & 1.5119 & 1.5115 & 1.4993 & 1.4997 & 1.5119 \\
\hline $\mathrm{H}_{s}-\mathrm{C}-\mathrm{As}$ & 112.294 & 112.296 & 112.493 & 112.459 & 112.263 \\
\hline $\mathrm{H}-\mathrm{As}-\mathrm{C}$ & 95.657 & 95.721 & 95.721 & 95.725 & 95.726 \\
\hline $\mathrm{H}_{a}-\mathrm{C}-\mathrm{As}$ & 108.367 & 108.345 & 108.248 & 108.218 & 108.315 \\
\hline $\mathrm{H}_{s}-\mathrm{C}-\mathrm{As}-\mathrm{H}$ & -46.310 & -46.293 & -46.296 & -46.291 & -46.288 \\
\hline $\mathrm{H}_{a}-\mathrm{C}-\mathrm{As}_{\mathrm{s}} \mathrm{H}_{s}$ & 121.192 & 121.206 & 121.294 & 121.290 & 121.202 \\
\hline
\end{tabular}

${ }^{a}$ CCSD(T)_AE/pwCVQZ-PP + MP2_AE/pwCV5Z-PP - MP2_AE/pwCVQZ-PP

recorded at room temperature, and at a pressure of a few tens of $\mu$ bar. The measurement accuracy for a strong isolated line was estimated to be $0.15 \mathrm{MHz}$.

In the frequency range 150 to $660 \mathrm{GHz}$, the spectrum of methylarsine was recorded using the Lille spectrometer [8], equipped with a fast-scan mode 9]. The measurements were performed in a static mode, in which the absorption cell was filled with the sample every 4 hours at typical pressure of $25-30 \mu$ bar. In the case of the Lille measurements, the measurement accuracy for a strong isolated line was estimated to be $0.05 \mathrm{MHz}$. This value is somewhat worse compared with the typical measurement accuracy of $0.03 \mathrm{MHz}$ and is chosen to take into account the partially resolved or unresolved hyperfine and internal rotation splittings that may lead to a broadening or a deformation of the lineshape even for a strong isolated line.

\section{Analysis}

\subsection{Quantum chemical calculations}

The structure calculations were carried out at the correlated levels of second-order Møller-Plesset perturbation theory (MP2) [10] and coupled-cluster theory with single and double excitations [11] augmented by a perturbational estimate of the effects of connected triple excitations $(\operatorname{CCSD}(\mathrm{T}))$ [12. The cc-pwCnZ-PP $(n=\mathrm{T}, \mathrm{Q}, 5)$ basis sets were used [13] all electrons being correlated (AE). The CCSD(T) calculations were performed with the MOLPRO program [14, 15] and the MP2 calculations with Gaussian 09, Revision D.01 [16].

Table 1 shows the equilibrium geometries computed at different levels of theory. Improvement of the basis from cc-pwCVTZ-PP to cc-pwCVQZ-PP leads to very small changes of the structural parameters except for the $\mathrm{C}-\mathrm{As}$ bond length which is shortened by as much as $0.004 \AA$. Therefore, the question arises whether the computed geometrical parameters of $\mathrm{CH}_{3} \mathrm{AsH}_{2}$ are already converged when using the cc-pwCVQZ-PP. For this reason, the structural effects of further basis set improvement (cc-pwCVQZ-PP $\rightarrow$ cc-pwCV5Z-PP) were also investigated. As the changes are expected to be small, the corrections were estimated at the MP2 level. The decrease for the $\mathrm{C}-\mathrm{As}$ bond length is small, $0.0007 \AA$ whereas the other parameters are not significantly affected. However, it is known that at the $\operatorname{CCSD}(\mathrm{T}) \_\mathrm{AE}$ level of theory with a quadruple zeta basis set, the cancellation of errors is optimum. This is no longer true for a larger basis set. To check this point, we have compared the rotational constants computed with the ab initio structure with the semiexperimental equilibrium ones. For this goal, the cubic force field has been calculated at two different levels of theory: the B3LYP method [17 with the pc3 basis set 18 and the MP2_FC method with the SDB-cc-pVTZ basis set [19. FC meaning that the frozen core approximation is used. The choice of this second method is justified by the fact that it delivers structures close to equilibrium values [20]. The derived rovibrational corrections are (in MHz): $B_{e}-B_{0}=83.08, C_{e}-C_{0}=82.82$ at the MP2_FC/SDB-cc-pVTZ level and $B_{e}-B_{0}=83.81, C_{e}-C_{0}=83.19$ at the $\mathrm{B} 3 \mathrm{LYP} / \mathrm{pc} 3$ level, respectively. As the $A$ rotational constant is much larger, its rovibrational correction is proportionally larger, and the agreement between different levels 
of theory is worse: $A_{e}-A_{0}=618.9 \mathrm{MHz}$ at the MP2_FC/SDB-cc-pVTZ level and $A_{e}-A_{0}=524.4 \mathrm{MHz}$ at the $\mathrm{B} 3 \mathrm{LYP} / \mathrm{pc} 3$ level (the $A / B$ ratio is about 7.4 in $\mathrm{CH}_{3} \mathrm{AsH}_{2}$ ). The two force fields give compatible values for $B_{e}-B_{0}$ and $C_{e}-C_{0}$ giving us confidence that they are reliable. It allows us to estimate the semiexperimental equilibrium rotational constants $B_{e}$ and $C_{e}$. Assuming that the CCSD(T)_AE/wCVQZ$\mathrm{PP}$ structure is accurate except for the $\mathrm{C}-\mathrm{As}$ bond length, it is possible to estimate this bond length by comparing the semiexperimental equilibrium rotational constants with the computed ones. Both $B_{e}$ and $C_{e}$ yield exactly the same value for $r_{e}(\mathrm{C}-\mathrm{As})=1.9686 \AA$. It is still possible to improve this value by applying an offset correction for the As- $\mathrm{H}$ bond length which may be obtained by comparing the ab initio and experimentally determined equilibrium values for $r(\mathrm{As}-\mathrm{H})$ in $\mathrm{AsH}_{3}$ [21. The structure of $\mathrm{AsH}_{3}$ computed at the $\operatorname{CCSD}(\mathrm{T}) \_\mathrm{AE} / \mathrm{pwCVQZ}-\mathrm{PP}$ level gives $r(\mathrm{As}-\mathrm{H})=1.5092 \AA$ whereas the experimental equilibrium value is 1.51130(16) $\AA$ 21. In other words, the $\operatorname{CCSD}(\mathrm{T}) \_\mathrm{AE} / \mathrm{pwCVQZ}-\mathrm{PP}$ value for As $-\mathrm{H}$ is too short by $0.0021 \AA$. In this particular case, the "offset" method gives reliable results because the As-H bond length in both molecules $\left(\mathrm{AsH}_{3}\right.$ and $\left.\mathrm{CH}_{3} \mathrm{AsH}_{2}\right)$ is quite similar as confirmed by using the Atom In Molecule (AIM) theory [22, see Appendix. Taking this small offset into account, it gives for $\mathrm{CH}_{3} \mathrm{AsH}_{2} r(\mathrm{As}-\mathrm{H})=1.5136 \AA$. Using this improved value of $r(\mathrm{As}-\mathrm{H})$, both $B_{e}$ and $C_{e}$ give $1.967 \AA$, confirming the hypothesis that it is better not to take into account the correction cc-pwCVQZ-PP $\rightarrow$ cc-pwCV5Z-PP.

For the prediction of the ground state rotational constants and torsional parameters, we retained the MP2/SDB-cc-pVTZ as well as DFT M06-2X/pc-4 method/basis set combinations. The M06-2X method [23] has previously been shown to provide relatively highly accurate results when compared with the experimental values 24] while demanding much less computational effort. In terms of rotational constants, the M06$2 \mathrm{X} / \mathrm{pc}-4$ calculation is the most satisfactory but only the MP2/SDB-cc-pVTZ 25] method in the frozen core approximation gives a geometry very close to the equilibrium structure. This behavior was previously noticed 25] and might be useful to calculate reliable structures of arsenic derivatives.

\subsection{Theoretical model}

The Hamiltonian used in the present work is the so-called RAM torsion-rotation Hamiltonian based on the works [26, 27], and [28, which has proved its effectiveness for a number of molecules containing the $C_{3 v}$ rotor and $C_{s}, C_{2 v}$, or $C_{1}$ molecular frames. In the current study we employ the RAM36 (rhoaxis-method for 3 and 6 -fold barriers) code that realizes the RAM approach for molecules with a $C_{3 v}$ top attached to a molecular frame of $C_{s}$ or $C_{2 v}$ symmetry and having a 3- or 6-fold barrier to internal rotation, respectively [29, 30. This code was successfully applied to molecules such as acetaldehyde [31 and methyl mercaptan 32 which like methylarsine are characterized by a rather large coupling between internal and overall rotations $\left(\rho=0.329\right.$ for $\mathrm{CH}_{3} \mathrm{CHO}, \rho=0.652$ for $\left.\mathrm{CH}_{3} \mathrm{SH}\right)$. A general expression for the RAM Hamiltonian implemented in the RAM36 code takes the following form:

$$
\begin{aligned}
H= & \frac{1}{2} \sum_{k n p q r s} B_{k n p q r s 0}\left[J^{2 k} J_{z}^{n} J_{x}^{p} J_{y}^{q} p_{\alpha}^{r} \cos (3 s \alpha)\right. \\
& \left.+\cos (3 s \alpha) p_{\alpha}^{r} J_{y}^{q} J_{x}^{p} J_{z}^{n} J^{2 k}\right]+ \\
& \frac{1}{2} \sum_{k n p q r t} B_{k n p q r 0 t}\left[J^{2 k} J_{z}^{n} J_{x}^{p} J_{y}^{q} p_{\alpha}^{r} \sin (3 t \alpha)\right. \\
& \left.+\sin (3 t \alpha) p_{\alpha}^{r} J_{y}^{q} J_{x}^{p} J_{z}^{n} J^{2 k}\right]
\end{aligned}
$$

where the $B_{\text {knpqrst }}$ are fitting parameters. In the case of a $C_{3 v}$ top and $C_{s}$ frame (as is appropriate for methylarsine), the allowed terms in the torsion-rotation Hamiltonian must be totally symmetric in the group $G_{6}$ (and also must be Hermitian and invariant to the time reversal operation).

To take the quadrupole hyperfine structure into account, we use a modified version of RAM36, which we call here RAM36hf, that allows us to fit together individual hyperfine components as well as torsion-rotation transitions with an unresolved hyperfine structure. Previously, the RAM36hf code was successfully applied to the analysis of the rotational spectrum of N-methylformamide [5] and nitromethane [6]. In the RAM36hf code the standard hyperfine energy expression is used: 


$$
\begin{aligned}
E_{h f} & =\left[\chi_{a a}\left\langle J_{a}^{2}\right\rangle+\chi_{c c}\left\langle J_{c}^{2}\right\rangle-\left(\chi_{a a}+\chi_{c c}\right)\left\langle J_{b}^{2}\right\rangle\right. \\
& \left.+\chi_{a c}\left\langle J_{a} J_{c}+J_{c} J_{a}\right\rangle\right] \frac{2 f(I, J, F)}{J(J+1)}
\end{aligned}
$$

where $f(I, J, F)$ is the Casimir function. In RAM36hf, the hyperfine structure and the fine torsion-rotation structure of the spectrum are treated simultaneously, in contrast to the approach applied in some other studies where hyperfine shifts are removed and hypothetical hyperfine free transition frequencies are fit using theoretical models that only take large amplitude motions in the molecule into account (as for example acetamide [33, 34] or methylamine [35, 36]).

\subsection{Assignment and fit}

Methylarsine is a prolate asymmetric top molecule close to the symmetric top limit. For a $C_{s}$ symmetry molecule, the RAM36hf code defines the symmetry plane as the $z x$ plane (molecule fixed-axis system). In the case of methylarsine, the symmetry plane is defined as the $a c$ plane (principal axis system). Therefore, in this study the molecular Hamiltonian was established in a rather unusual $I^{l}$ coordinate representation: $z=a, x=c$, and $y=b$.

We started the assignment with the cm-wave spectra where the transition $1_{0,1} \leftarrow 0_{0,0}$ in the ground vibrational state was easily located around $17.6 \mathrm{GHz}$ on the basis of the predictions obtained from quantum chemical calculations. In the vicinity of the ground state transition, we also assigned the transition of the first excited torsional state which is characterized by a larger torsional $A-E$ splitting and a lower intensity owing to the lower Boltzmann factor. In the next step, we assigned a series of $a$-type transitions with $J=2 \leftarrow 1,3 \leftarrow 2$, and $4 \leftarrow 3$. The initial fit of these transitions allowed us to make predictions which were good enough to continue the assignment. The assignment was continued in the classical bootstrap manner, where newly assigned transitions were used to improve the frequency predictions and search for new ones. In total, we assigned 2473 distinct frequency lines corresponding to 4158 transitions of the ground, first, and second excited torsional states with the $J$ quantum number up to 50 . In addition to $a$-type transitions, we also assigned $c$-type transitions owing to non-zero $\mu_{c}$ dipole moment component. In general, the calculation of the dipole moment using different methods (MP2, B3LYP, MO62X) and different basis sets (wCV5Z-PP, pc3, pc4) gives consistent results with $0.84 \leq \mu_{a} \leq 0.88 \mathrm{D}$ (in agreement with ref. [3]) and $0.22 \leq \mu_{c} \leq 0.32 \mathrm{D}$. The $\mu_{b}$ dipole moment component is set to zero due to molecular symmetry. The assigned transitions were fitted using the RAM36hf code to the RAM Hamiltonian model containing 45 parameters plus 3 quadrupole hyperfine parameters $\chi_{a a}$, $\chi_{c c}$ and off-diagonal $\chi_{a c}$. A fit achieving a unitless rms deviation of 0.92 was obtained. The final set of Xacecular parameters is presented in Table 2. The numbers of terms in the model distributed between the $n_{o p}=2,4,6,8$ orders of the operators are equal to or less than the total numbers of determinable parameters for those orders, as calculated according the ordering scheme of 37. We note in passing that the ordering scheme of [37] assumes that $\cos (3 n \alpha)$ and $\sin (3 n \alpha)$ are regarded as $2 n^{\text {th }}$-order terms, whereas $\left(p_{\alpha}\right)^{n}$ and $\left(J_{i}\right)^{n}(i=x, y, z)$ are regarded as $n^{t h}$-order terms. There were no problems with convergence observed for the final fit. The full dataset of the assigned rotational transitions of methylarsine is available as a Supplementary material. The table provided in Supplementary material includes for each transition: assignment, measured (observed) frequency and its uncertainty, calculated frequency and its uncertainty, as well as 'obs-calc' difference obtained in the final fit.

A portion of the rotational spectrum of methylarsine predicted using the final set of molecular parameters is shown on Fig. 1 where it is compared with the experimental spectrum. The overall correspondence between the experimental and theoretical spectra is good. The strongest lines are well predicted by our current model, although one can also find several relatively strong unassigned lines around $245 \mathrm{GHz}$. These lines correspond to rotational transitions of the third excited torsional state that are predicted by our model sufficiently well to be assigned. However they are not included in the current fit, as we suppose that the $v_{t}=3$ state may be subjected to a downward propagation of a perturbation of the $\nu_{9}$ mode, which is located in vicinity of 
Table 2: Molecular parameters of methylarsine obtained with the RAM36HF program.

\begin{tabular}{|c|c|c|c|}
\hline \multicolumn{3}{|c|}{$n t r^{\mathrm{a}}$ Parameter $^{\mathrm{b}}$ Operator $^{\mathrm{c}}$} & Value $^{\mathrm{d}}$ \\
\hline 220 & $F$ & $p_{\alpha}^{2}$ & $8.95757(18)$ \\
\hline 220 & $V_{3}$ & $\frac{1}{2}(1-\cos 3 \alpha)$ & $517.515(14)$ \\
\hline 211 & $\rho$ & $J_{z} p_{\alpha}$ & $0.41295517(25)$ \\
\hline 202 & $A_{R A M}$ & $J_{z}^{2}$ & $2.175536156(93)$ \\
\hline 202 & $B_{R A M}$ & $J_{y}^{2}$ & $0.294896600(32)$ \\
\hline 202 & $C_{R A M}$ & $J_{x}^{2}$ & $0.293565870(59)$ \\
\hline 202 & $2 D_{z x}$ & $\frac{1}{2}\left\{J_{z}, J_{x}\right\}$ & $-0.11038(23) \times 10^{-1}$ \\
\hline 440 & $V_{6}$ & $\frac{1}{2}(1-\cos 6 \alpha)$ & $-3.2752(12)$ \\
\hline 431 & $\rho_{m}$ & $J_{z} p_{\alpha}^{3}$ & $-0.4798(50) \times 10^{-4}$ \\
\hline 422 & $F_{J}$ & $J^{2} p_{\alpha}^{2}$ & $-0.126810(39) \times 10^{-4}$ \\
\hline 422 & $F_{K}$ & $J_{z}^{2} p_{\alpha}^{2}$ & $-0.13706(63) \times 10^{-3}$ \\
\hline 422 & $F_{x y}$ & $p_{\alpha}^{2}\left(J_{x}^{2}-J_{y}^{2}\right)$ & $0.7755(63) \times 10^{-6}$ \\
\hline 422 & $V_{3 J}$ & $J^{2}(1-\cos 3 \alpha)$ & $-0.1755142(64) \times 10^{-2}$ \\
\hline 422 & $V_{3 K}$ & $J_{z}^{2}(1-\cos 3 \alpha)$ & $0.54448(21) \times 10^{-2}$ \\
\hline 422 & $V_{3 z x}$ & $\frac{1}{2}(1-\cos 3 \alpha)\left\{J_{z}, J_{x}\right\}$ & $0.32268(81) \times 10^{-2}$ \\
\hline 422 & $V_{3 x y}$ & $\left(J_{x}^{2}-J_{y}^{2}\right)(1-\cos 3 \alpha)$ & $0.29041(51) \times 10^{-4}$ \\
\hline 422 & $D_{3 z y}$ & $\frac{1}{2} \sin 3 \alpha\left\{J_{z}, J_{y}\right\}$ & $-0.811(20) \times 10^{-3}$ \\
\hline 413 & $\rho_{J}$ & $J^{2} J_{z} p_{\alpha}$ & $0.129344(40) \times 10^{-4}$ \\
\hline 413 & $\rho_{K}$ & $J_{z}^{3} p_{\alpha}$ & $0.14880(29) \times 10^{-3}$ \\
\hline 413 & $\rho_{x y}$ & $\frac{1}{2} p_{\alpha}\left\{J_{z}\right.$ & $-0.6897(76) \times 10^{-6}$ \\
\hline 404 & $D_{z x J}$ & $\frac{1}{2} J^{2}\left\{J_{z}, J_{x}\right\}$ & $0.4225(22) \times 10^{-7}$ \\
\hline 404 & $D_{J}$ & $-J^{4}$ & $0.282763(28) \times 10^{-6}$ \\
\hline 404 & $D_{J K}$ & $-J^{2} J_{z}^{2}$ & $0.45022(11) \times 10^{-5}$ \\
\hline 404 & $D_{K}$ & $-J_{z}^{4}$ & $0.42359(67) \times 10^{-4}$ \\
\hline 404 & $d_{1}$ & $J^{2}\left(J_{+}^{2}+J_{-}^{2}\right)$ & $0.19718(10) \times 10^{-8}$ \\
\hline 404 & $d_{2}$ & $J_{+}^{4}+J_{-}^{4}$ & $-0.3992(34) \times 10^{-9}$ \\
\hline 660 & $V_{9}$ & $\frac{1}{2}(1-\cos 9 \alpha)$ & $-0.33361(93)$ \\
\hline 642 & $V_{6 J}$ & $J^{2}(1-\cos 6 \alpha)$ & $0.300(14) \times 10^{-5}$ \\
\hline 642 & $V_{6 z x}$ & $\frac{1}{2}(1-\cos 6 \alpha)\left\{J_{z}, J_{x}\right\}$ & $0.467(13) \times 10^{-3}$ \\
\hline 642 & $V_{6 x y}$ & $(1-\cos 6 \alpha)\left(J_{x}^{2}-J_{y}^{2}\right)$ & $0.2609(77) \times 10^{-5}$ \\
\hline 633 & $\rho_{m J}$ & $J^{2} p_{\alpha}^{3} J_{z}$ & $0.1664(66) \times 10^{-8}$ \\
\hline 624 & $F_{J J}$ & $J^{4} p_{\alpha}^{2}$ & $0.2824(92) \times 10^{-10}$ \\
\hline 624 & $F_{J K}$ & $J^{2} J_{z}^{2} p_{\alpha}^{2}$ & $-0.1673(64) \times 10^{-8}$ \\
\hline 624 & $V_{3 J J}$ & $J^{4}(1-\cos 3 \alpha)$ & $0.1824(28) \times 10^{-8}$ \\
\hline 624 & $V_{3 J K}$ & $J^{2} J_{z}^{2}(1-\cos 3 \alpha)$ & $-0.5489(27) \times 10^{-7}$ \\
\hline 624 & $V_{3 K K}$ & $J_{z}^{4}(1-\cos 3 \alpha)$ & $0.1347(66) \times 10^{-6}$ \\
\hline 624 & $V_{3 x y J}$ & $J^{2}(1-\cos 3 \alpha)\left(J_{x}^{2}-J_{y}^{2}\right)$ & $-0.5259(86) \times 10^{-9}$ \\
\hline 624 & $V_{3 x y 4}$ & $\cos 3 \alpha\left(J_{x}^{4}+J_{y}^{4}\right)$ & $-0.665(35) \times 10^{-9}$ \\
\hline 615 & $\rho_{J J}$ & $J^{4} J_{z} p_{\alpha}$ & $-0.323(10) \times 10^{-10}$ \\
\hline 615 & $\rho_{J K}$ & $J^{2} J_{z}^{3} p_{\alpha}$ & $0.457(16) \times 10^{-9}$ \\
\hline 606 & $H_{J}$ & $J^{6}$ & $-0.1125(21) \times 10^{-12}$ \\
\hline 606 & $H_{J K}$ & $J^{4} J_{z}^{2}$ & $0.1211(33) \times 10^{-10}$ \\
\hline 862 & $V_{9 J}$ & $J^{2}(1-\cos 9 \alpha)$ & $0.2217(83) \times 10^{-5}$ \\
\hline 844 & $V_{6 J J}$ & $J^{4}(1-\cos 6 \alpha)$ & $0.191(10) \times 10^{-9}$ \\
\hline 844 & $V_{6 J K}$ & $J^{2} J_{z}^{2}(1-\cos 6 \alpha)$ & $-0.317(22) \times 10^{-8}$ \\
\hline & $\chi_{z z}$ & & $0.12040(22) \times 10^{-2}$ \\
\hline & $\chi_{x x}$ & & $-0.39315(14) \times 10^{-2}$ \\
\hline & $2 \chi_{z x}$ & & $0.769(22) \times 10^{-2}$ \\
\hline
\end{tabular}

${ }^{\text {a }} n=t+r$, where $n$ is the total order of the operator, $t$ is the order of the torsional part and $r$ is the order of the rotational part, respectively.

${ }^{\mathrm{b}}$ Parameter nomenclature based on the subscript procedures of Ref. 38.

c $\{A, B\}=A B+B A$. The product of the operator in the third column of a given row and the parameter in the second column of that row gives the term actually used in the torsion-rotation Hamiltonian of the program, except for $F, \rho$ and $A_{R A M}$, which occur in the Hamiltonian in the form $F\left(p_{\alpha}-\rho J_{z}\right)^{2}+A_{R A M} J_{z}^{2}$.

${ }^{\mathrm{d}}$ All values are in $\mathrm{cm}^{-1}$ (except $\rho$ which is unitless). Statistical uncertainties are shown as one standard uncertainty in the units of the last two digits. 
Figure 1: Predicted and calculated spectra of $\mathrm{CH}_{3} \mathrm{AsH}_{2}$ in the vicinity of the $J=14 \leftarrow 13$ series of ${ }^{a} R_{0,1}$ transitions of $v_{t}=0$, 1 , and 2 states.

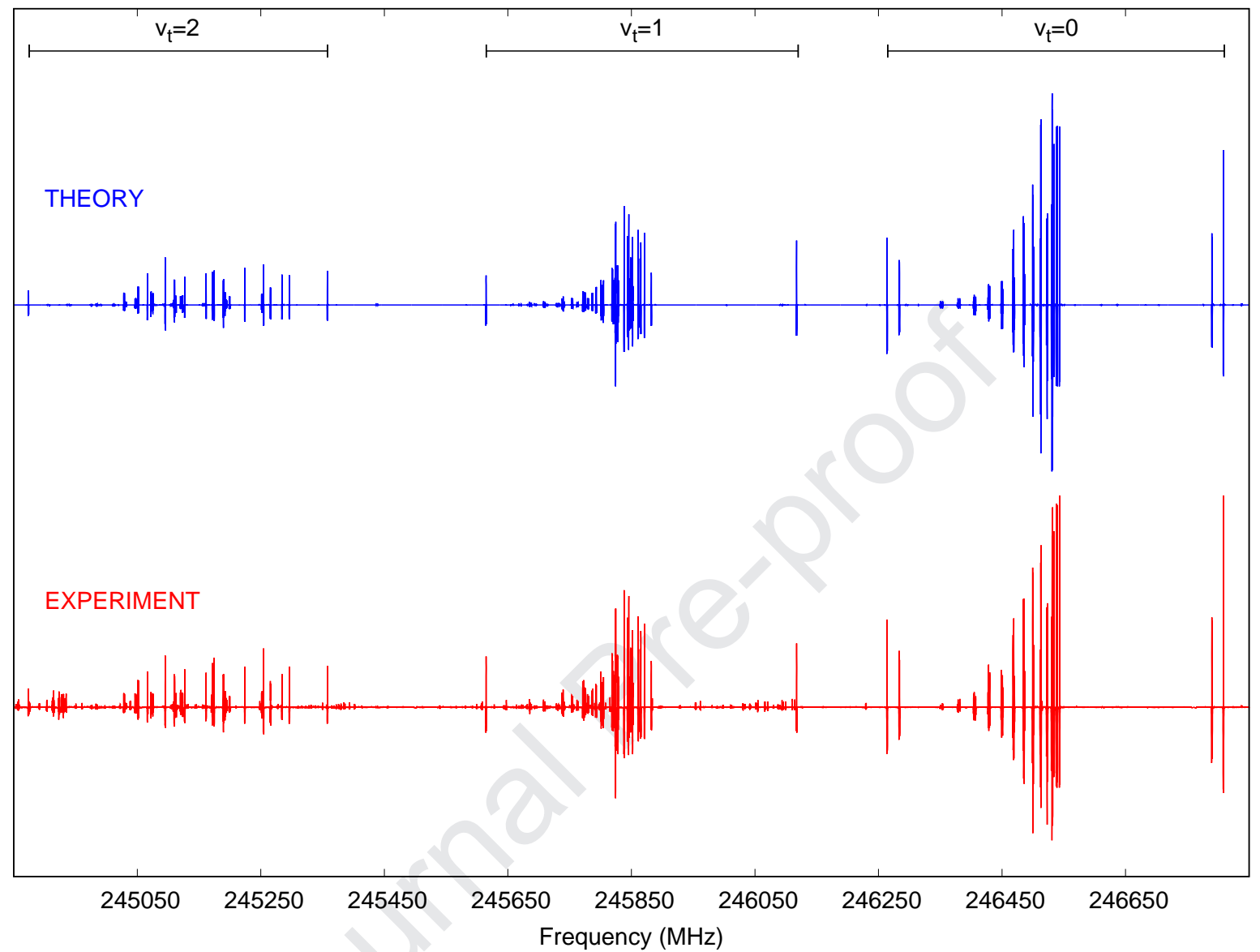

the $v_{t}=4$ torsional excited state. Such type of perturbation propagation was previously observed in the case of methyl mercaptan 32 .

An example of comparison of the observed and predicted spectra at a finer scale is shown on Fig. 2 Owing to the hyperfine structure and internal rotation, the spectrum contains a set of multiple components many of which are not resolved in the Doppler resolution mode of the spectrometer. A combination of resolved and unresolved components may form rather complex multiplets that depend on the interplay of quadrupole, asymmetry and torsional splittings in the spectrum. As was already mentioned above, the asymmetry splittings for some energy levels in methylarsine may have the same order of magnitude as the quadrupole splittings, that could affect the first order perturbation treatment of the hyperfine energies. Nevertheless, there were no 'anomalous' hyperfine splittings (i.e. deviating from the current model) observed in this study. It is seen that the final set of molecular parameters predicts well the experimental spectrum reported on Fig. 2

\section{Discussion}

\subsection{Internal rotation and centrifugal distortion parameters}

Table 3 presents a comparison of rotational, quartic centrifugal distortion, torsion, and quadrupole coupling parameters of methylarsine obtained from the RAM36hf fit and from quantum chemical calculations. 
Figure 2: A portion of a simulated stick spectrum of $\mathrm{CH}_{3} \mathrm{AsH}_{2}$ around $228930 \mathrm{MHz}$ with assignments of rotational transitions that contribute to this stick spectrum. Each stick corresponds to a hyperfine component of a torsion-rotation transition. In blue: line profile spectrum simulated using the stick spectrum. In red: experimental spectrum of $\mathrm{CH}_{3} \mathrm{AsH}_{2}$. For interpretation of the references to color in this figure legend the reader is referred to the web version of this article.

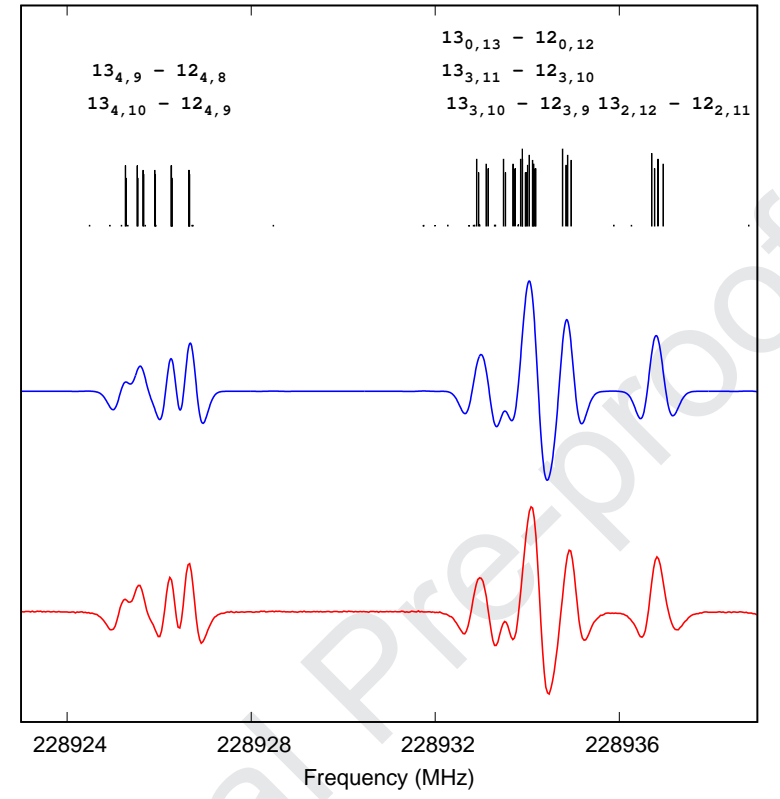

Table 3: Comparison of the main rotation, torsion and hyperfine parameters of methylarsine with ab initio results

\begin{tabular}{l|rr}
\hline Parameter & RAM36HF fit & M06-2X/pc-4 \\
\hline$A(\mathrm{MHz})$ & 65221.4 & 65641.6 \\
$B(\mathrm{MHz})$ & 8840.8 & 8840.2 \\
$C(\mathrm{MHz})$ & 8800.4 & 8793.0 \\
$D_{J}(\mathrm{kHz})$ & 8.477 & 8.151 \\
$D_{J K}(\mathrm{kHz})$ & 134.97 & 36.29 \\
$D_{K}(\mathrm{kHz})$ & 1269.9 & 202.6 \\
$d_{1}(\mathrm{kHz})$ & 0.05911 & 0.05591 \\
$d_{2}(\mathrm{kHz})$ & -0.01197 & -0.01118 \\
$F\left(\mathrm{~cm}^{-1}\right)$ & 8.958 & 9.051 \\
$V_{3}\left(\mathrm{~cm}^{-1}\right)$ & 517.5 & 482.5 \\
$\rho$ & 0.413 & 0.409 \\
$\chi_{a a}(\mathrm{MHz})$ & 35.4 & 34.7 \\
$\chi_{c c}(\mathrm{MHz})$ & -117.2 & -122.8 \\
$\chi_{a c}(\mathrm{MHz})$ & 115.7 & 119.8 \\
\hline \hline
\end{tabular}


As one can see, a good agreement is observed for all parameters except for two centrifugal distortion constants $D_{J K}$ and $D_{K}$. Initially we thought that this discrepancy is the consequence of the way the coupling of internal and overall rotations is treated in the RAM36 code. According footnote 'c' of Table 2 the second order parameters $F, \rho$ and $A_{\mathrm{RAM}}$ occur in the Hamiltonian in the form $F\left(p_{\alpha}-\rho J_{z}\right)^{2}+A_{\mathrm{RAM}} J_{z}^{2}$. The $F\left(p_{\alpha}-\rho J_{z}\right)^{2}+A_{\mathrm{RAM}} J_{z}^{2}$ part of the Hamiltonian may be regrouped (as it was done for example in Ref. 29 to reduce a correlation between main parameters) as $F\left(p_{\alpha}\right)^{2}-2 F \rho p_{\alpha} J_{z}+A_{\text {eff }} J_{z}^{2}$, where $A_{\text {eff }}=A_{\mathrm{RAM}}+F(\rho)^{2}$ and $F(\rho)^{2}$ is a modification of the rotational constant $A$ due to internal rotation. The $F(\rho)^{2}$ modification is negligible when the $\rho$ value is small, but is very significant $\left(1.52 \mathrm{~cm}^{-1}\right)$ in methylarsine where the $\rho$ value is rather large (0.413). In the RAM36 code, the mixed internal angular momentum expression $p_{\alpha}-\rho J_{z}$ which brings together the coupling between internal and overall rotation is used only in the second order of the RAM Hamiltonian. This gives an unbiased $A$ rotational constant which can be compared to the ab initio value after transforming from RAM to PAM axis system (and we see in Table 3 a rather good agreement for this constant). For the higher orders of the RAM Hamiltonian (fourth, six etc.), RAM36 does not allow at the moment a mixed angular momentum expression $p_{\alpha}-\rho J_{z}$ and all corresponding terms only contain powers of the internal rotation angular momentum $p_{\alpha}$. Thus, we expected some modifications of the values of centrifugal distortion parameters caused by the fact that the coupling between internal and overall rotations is not represented explicitly in the 4th, 6th etc. orders of torsion-rotation RAM Hamiltonian terms.

One may think that a way to estimate these modifications of the centrifugal distortion parameters due to the non-explicit way of representing the coupling between internal and overall rotations at higher orders of the RAM Hamiltonian would be to replace $p_{\alpha}$ by the mixed internal angular momentum expression $p_{\alpha}-\rho J_{z}$ in the RAM Hamiltonian of Table 2, and after raising to corresponding powers and opening brackets collect those terms which will contribute to $D_{K}, D_{J K}$ etc. as it was done above for the $A$ rotational constant. In our case, this would mean that from the available Hamiltonian terms of Table 2 we need to take the contributions due to $\rho_{K}, \rho_{m}, F_{K}$ into account in the case of $D_{K}$, and $F_{J}, \rho_{J}$ in the case of $D_{J K}$. In practice, this procedure does not work because the replacement of $p_{\alpha}$ by the mixed internal angular momentum expression $p_{\alpha}-\rho J_{z}$ led to a significant change in the values of all parameters of corresponding order. If we consider for example the $F_{m}$ term (not present in the model of Table 2), and replace $\left(p_{\alpha}\right)^{4}$ by $\left(p_{\alpha}-\rho J_{z}\right)^{4}$ with further expanding the obtained expression we will see that the transformed $F_{m}$ term in addition to $\left(p_{\alpha}\right)^{4}$ term itself also contains $p_{\alpha}^{3} J_{z}, p_{\alpha}^{2} J_{z}^{2}, p_{\alpha} J_{z}^{3}$, and $J_{z}^{4}$ terms which are related to the $\rho_{m}, F_{K}, \rho_{K}$, and $D_{K}$ parameters of the original model of Table 2.

$$
H_{\mathrm{TR}, \text { mixed }}^{(m)}=F_{m}^{*}\left(p_{\alpha}^{4}-4 \rho p_{\alpha}^{3} J_{z}+6 \rho^{2} p_{\alpha}^{2} J_{z}^{2}-4 \rho^{3} p_{\alpha} J_{z}^{3}+\rho^{4} J_{z}^{4}\right)
$$

Similarly, if we perform the same procedure of replacing $p_{a}$ by $p_{a}-\rho J_{z}$ for $F_{J}, F_{K}$ terms we will see that after the transformation they will contain terms not only related to the $D_{J K}, D_{K}$ parameters but also to the $\rho_{J}$, and $\rho_{K}$ parameters:

$$
\begin{gathered}
H_{\mathrm{TR}, \text { mixed }}^{(J)}=F_{J}^{*}\left(p_{\alpha}^{2} J^{2}-2 \rho p_{\alpha} J_{z} J^{2}+\rho^{2} J_{z}^{2} J^{2}\right) \\
H_{\mathrm{TR}, \text { mixed }}^{(K)}=F_{K}^{*}\left(p_{\alpha}^{2} J_{z}^{2}-2 \rho p_{\alpha} J_{z}^{3}+\rho^{2} J_{z}^{4}\right)
\end{gathered}
$$

Because of this interconnection between different terms, the calculation of the modifications of centrifugal distortion parameters due to the coupling of internal and overall rotations based on the parameter values of Table 2 is not that straightforward.

A way to get an estimate of the centrifugal distortion constants unbiased by the torsion-rotation coupling would be to perform a fit with $p_{\alpha}$ replaced by the mixed internal angular momentum expression $p_{\alpha}-\rho J_{z}$ in all 4th order terms containing $p_{\alpha}$. Unfortunately, at the moment the RAM36 code does allow such fit. What we can try to do is to represent the terms with mixed internal angular momentum expressions by a linear combination of terms allowed by RAM36, i.e. implement equations Eqs. 34 as a linear combination of terms with fixed ratio coefficients calculated from the current $\rho$ value. The coefficients in front of these linear combinations i.e. $F_{J}^{*}, F_{K}^{*}$, etc. will be varied by RAM36 whereas the ratios inside these combinations (which depend on different powers of $\rho$ ) will be fixed. The star symbol is used here to distinguish between the parameters of the operators in Table 2, and the parameters of the linear combination terms of Eqs. 34.4 
When trying to determine the $D_{J K}$ and $D_{K}$ constants without torsional contribution, we performed a new least-squares fit in which we replaced all 4 th order parameters containing $p_{\alpha}$ namely $F_{J}, F_{K}, F_{b c}, \rho_{J}, \rho_{K}, \rho_{b c}$, and $\rho_{m}$, from the Hamiltonian model of Table 2 by a group of terms $F_{J}^{*}, F_{K}^{*}$, etc. representing expressions analogous to Eqs. 34. In this case we got practically the same rms deviation of the fit, but the values of 4 th order torsion-rotation centrifugal distortion parameters are changed, redistributing contributions to energy levels between new versions of the terms. As a result of such a redistribution we got a much better agreement for the $D_{J K}$ parameter (transformed value $39.6708(24) \mathrm{kHz}$ ) but for the $D_{K}$ parameter the deviation from the quantum chemical calculations value became much larger (the transformed value of $72082 .(34) \mathrm{kHz}$ instead of $1269.9 \mathrm{kHz}$ of Table 2 with quantum chemical calculations value being $202.49 \mathrm{kHz}$ ). Such a large discrepancy cannot be explained by the rotation from the RAM to PAM axis systems since the angle between those two systems is only 0.17 degree and even for the $A$ rotation constant the correction due to rotation from RAM to PAM is only $0.5 \mathrm{MHz}$ or $7.7 \times 10^{-4} \%$.

Two possible explanations may be considered. The first one is connected to some strong intervibrational interaction with a low lying nontorsional mode. This perturbation may propagate through intertorsional interactions down to the torsional states considered in this study and may be absorbed in part by the $F_{K}$, $\rho_{K}$, and $D_{K}$ parameters (namely replacing $p_{\alpha}$ by $p_{\alpha}-\rho J_{z}$ in the $F_{K}, \rho_{K}$ terms causes this huge increase in the $D_{K}$ value when we try to take explicitly into account the contribution from the torsion-rotation coupling). The second possible explanation is connected to the reduction scheme of the Hamiltonian, which we effectively adopt by keeping one set or the other one of the RAM Hamiltonian parameters. According to the ordering scheme of [37] we can have 22 parameters for the 4th order RAM Hamiltonian as calculated from the difference between the total number of symmetry-allowed Hamiltonian terms of 4th order (33) and the number of symmetry-allowed contact transformation terms of 3rd order (11). The problems may appear if one tries to eliminate one term with a relatively large magnitude (as calculated from the molecular force field), keeping another term of relatively small magnitude. Then, a contribution to the energy from the eliminated 'large' term should be somehow redistributed among other terms. If by misfortune, at the

260 beginning of our fitting process for the methylarsine spectrum, we don't include some important term whose contribution is then effectively redistributed between other terms of 4 th and higher orders, we may believe that this term is insignificant (since we have a compensation of its contribution from other terms) but the values of some other parameters appear to be biased. Considerable work should be done before we will be able to check both these explanations of the discrepancy of $D_{K}$ value.

\subsection{Tunneling barriers}

Using the $V_{3}, V_{6}$, and $V_{9}$ terms of the potential energy function from Table 2, the estimated value of the barrier to internal rotation in methylarsine is $513.84 \mathrm{~cm}^{-1}$. It is somewhat higher compared to the value obtained from the M06-2X/pc-4 calculations in Table 3 indicating that while this method/basis set combination gives excellent consistency for the experimental rotational constants, it is less suited for torsional barrier height estimation in the case of methylarsine. At the same time, the obtained experimental value of the barrier height is in excellent agreement with the theoretical value of $518.3 \mathrm{~cm}^{-1}$ obtained by Kim et al. 39 using a fitted potential energy surface generated from the optimized geometries of methylarsine. Moreover, in the present study, the estimated energies of the $0_{0,0}$ rotational levels of $A$ and $275 E$ symmetry of the $v_{t}=1$ state are respectively $181.1 \mathrm{~cm}^{-1}$ and $179.4 \mathrm{~cm}^{-1}$. These values are also in agreement with the frequency of the torsional mode of $185 \mathbf{c m}^{-1}$ measured in the low-resolution IR spectrum of methylarsine by Harvey and Wilson [4], and determined by the theoretical calculations by Kim et al. [39]. In ref. [39], torsion barrier and inversion barrier of the first and second-order for the $\mathrm{CH}_{3} \mathrm{XH}_{2}(\mathrm{X}=\mathrm{N}, \mathrm{P}, \mathrm{As}, \mathrm{Sb})$ molecules are compared.

280 In particular, in ref. [39] it is shown that the first and second-order inversion barriers exhibit a rapid increase from $\mathrm{CH}_{3} \mathrm{NH}_{2}\left(22.8\right.$ and $22.41 \mathrm{~kJ} / \mathrm{mol}$ respectively) to $\mathrm{CH}_{3} \mathrm{PH}_{2}(149.88$ and $149.65 \mathrm{~kJ} / \mathrm{mol}$ respectively), $\mathrm{CH}_{3} \mathrm{AsH}_{2}(170.93 \mathrm{~kJ} / \mathrm{mol}$ and $170.70 \mathrm{~kJ} / \mathrm{mol})$, and $\mathrm{CH}_{3} \mathrm{SbH}_{2}$ (204.44 and $204.24 \mathrm{~kJ} / \mathrm{mol}$ respectively). In contrast, the torsional barriers show only a slight decline from 8.73 for $\mathrm{CH}_{3} \mathrm{NH}_{2}$ to $5.53 \mathrm{~kJ} / \mathrm{mol}$ for $\mathrm{CH}_{3} \mathrm{AsH}_{2}$, and to $3.06 \mathrm{~kJ} / \mathrm{mol}$ for $\mathrm{CH}_{3} \mathrm{SbH}_{2}$. Therefore, both torsional and inversion splittings were observed in the rotational 
spectrum of methylamine [36] but only torsional splittings were observed in the rotational spectra of methylphosphine [40], and methylarsine (this study). In the latter two cases, owing to high barriers, the inversion splittings are so small that they were not resolved under the Doppler-limited spectral resolution of conventional absorption spectrometers used to record the spectra.

\section{Conclusion}

We performed a new study of the methylarsine spectrum in a broad frequency range, from 15 to 660 GHz. The new data for the $v_{t}=0,1,2$ torsional states involving rotational transitions with $J$ up to 50 were analyzed using the rho-axis-method and a fit within experimental error has been achieved. There were no 'anomalous' hyperfine splittings (i.e. deviating from the current model) observed in this study of methylarsine spectrum, although the asymmetry and quadrupole splittings for some energy levels have the same order of magnitude, that in principle could affect the first order perturbation treatment of the hyperfine energies employed in this study. A comparison of the obtained results with high level ab initio calculations of the quartic centrifugal distortion parameters reveals a significant discrepancy in the value of the $D_{K}$ parameter, which we are not able to explain at the current stage of methylarsine spectrum investigation.

\section{Acknowledgements}

Financial support by the Dr. Barbara Mez-Starck Foundation (Germany) is gratefully acknowledged. J.-C.G. thanks the Centre National d'Etudes Spatiales (CNES) for a grant.

\section{Appendix A.}

The Atom in Molecules (AIM) theory [22, 41] with its implementation in Gaussian by Cioslowski et al. [42, 43, 44, 45, 46, 47, 48, was used. The calculations were performed at the B3LYP/6-311+G(2d,2p) level of theory with the equilibrium structure. The following parameters were compared:

- The bond critical point density, $\rho_{b}$, which is a measure of the amount of electron density shared between the two bonded atoms.

- The bond ellipticity, $\epsilon$. It provides a measure of the extent to which the charge is preferentially accumulated at a given angle in a plane perpendicular to the bond path.

- The charges, $q$, on the atoms.

The strength of a bond increases and its length decreases as the bond critical point density $\rho_{b}$ increases. The increasing charges on the bonded atoms have the same effect.

Table A.4: Bond critical point densities, $\rho_{b}$ (a.u.), bond ellipticies, $\epsilon$, and charges $q$ (a.u.) on the atoms for the As-H bond calculated at the B3LYP $6-311+\mathrm{G}(2 \mathrm{~d}, 2 \mathrm{p})$

\begin{tabular}{lll} 
& $\mathrm{AsH}_{3}$ & $\mathrm{CH}_{3} \mathrm{AsH}_{2}$ \\
\hline$\rho_{b}$ & 0.1505 & 0.1505 \\
$\epsilon$ & 0.034 & 0.039 \\
$q(\mathrm{As})$ & 0.831 & 0.834 \\
$q(\mathrm{H})$ & -0.277 & -0.277 \\
\hline \hline
\end{tabular}


[12] K. Raghavachari, G. W. Trucks, J. A. Pople, M. Head-Gordon, A fifth-order perturbation comparison of electron correlation theories, Chem. Phys. Lett. 157 (1989) 479-483.

[13] K. A. Peterson, Systematically convergent basis sets with relativistic pseudopotentials. I. Correlation consistent basis sets for the post-d group 13-15 elements, J. Chem. Phys. 119 (2003) 11099-11112.

[14] H.-J. Werner, P. J. Knowles, G. Knizia, F. R. Manby, M. Schütz, Molpro: a general-purpose quantum chemistry program package, WIREs Comput Mol Sci 2 (2012) 242-253.

[15] H.-J. Werner, P. J. Knowles, G. Knizia, F. R. Manby, M. Schütz, P. Celani, W. Györffy, D. Kats, T. Korona, R. Lindh, A. Mitrushenkov, G. Rauhut, K. R. Shamasundar, T. B. Adler, R. D. Amos, S. J. Bennie, A. Bernhardsson, A. Berning, D. L. Cooper, M. J. O. Deegan, A. J. Dobbyn, F. Eckert, E. Goll, C. Hampel, A. Hesselmann, G. Hetzer, T. Hrenar, G. Jansen, C. Köppl, S. J. R. Lee, Y. Liu, A. W. Lloyd, Q. Ma, R. A. Mata, A. J. May, S. J. McNicholas, W. Meyer, T. F. Miller III, M. E. Mura, A. Nicklass, D. P. O’Neill, P. Palmieri, D. Peng, K. Pflüger, R. Pitzer, M. Reiher, T. Shiozaki, H. Stoll, A. J. Stone, R. Tarroni, T. Thorsteinsson, M. Wang, M. Welborn, MOLPRO, version 2019.2, a package of ab initio programs, see https://www.molpro.net (2019).

[16] M. J. Frisch, G. W. Trucks, H. B. Schlegel, G. E. Scuseria, M. A. Robb, J. R. Cheeseman, G. Scalmani, V. Barone, B. Mennucci, G. A. Petersson, H. Nakatsuji, M. Caricato, X. Li, H. P. Hratchian, A. F. Izmaylov, J. Bloino, G. Zheng, J. L. Sonnenberg, M. Hada, M. Ehara, K. Toyota, R. Fukuda, J. Hasegawa, M. Ishida, T. Nakajima, Y. Honda, O. Kitao, H. Nakai, T. Vreven, J. A. Montgomery, Jr., J. E. Peralta, F. Ogliaro, M. Bearpark, J. J. Heyd, E. Brothers, K. N. Kudin, V. N. Staroverov, R. Kobayashi, J. Normand, K. Raghavachari, A. Rendell, J. C. Burant, S. S. Iyengar, J. Tomasi, M. Cossi, N. Rega, J. M. Millam, M. Klene, J. E. Knox, J. B. Cross, V. Bakken, C. Adamo, J. Jaramillo, R. Gomperts, R. E. Stratmann, O. Yazyev, A. J. Austin, R. Cammi, C. Pomelli, J. W. Ochterski, R. L. Martin, K. Morokuma, V. G. Zakrzewski, G. A. Voth, P. Salvador, J. J. Dannenberg, S. Dapprich, A. D. Daniels, O. Farkas, J. B. Foresman, J. V. Ortiz, J. Cioslowski, D. J. Fox, Gaussian 09 Revision D.01, gaussian Inc. Wallingford CT 2009.

[17] C. Lee, W. Yang, R. Parr, Development of the Colle-Salvetti conelation energy formula into a functional of the electron density, Phys. Rev. B 37 (1988) 785-789.

[18] F. Jensen, Polarization consistent basis sets. VII. The elements K, Ca, Ga, Ge, As, Se, Br, and Kr, J. Chem. Phys. 136 (2012) 114107

[19] J. M. Martin, A. Sundermann, Correlation consistent valence basis sets for use with the Stuttgart-Dresden-Bonn relativistic effective core potentials: The atoms Ga-Kr and In-Xe, J. Chem. Phys. 114 (2001) 3408-3420.

[20] A. G. Császár, Anharmonic molecular force fields, Wiley Interdisciplinary Reviews: Computational Molecular Science 2 (2012) 273-289.

[21] O. N. Ulenikov, E. S. Bekhtereva, Y. B. Yuhnik, H. Bürger, High-resolution infrared study of the $\nu_{1}$ and $\nu_{3}$ bands, and the equilibrium structure of $\mathrm{AsD}_{3}$, J. Mol. Struct. 780 (2006) 115-123.

[22] R. Bader, Atoms in molecules: A quantum theory, Clarendon Press (1990).

[23] Y. Zhao, D. G. Truhlar, The M06 suite of density functionals for main group thermochemistry, thermochemical kinetics, noncovalent interactions, excited states, and transition elements: two new functionals and systematic testing of four M06-class functionals and 12 other functionals, Theor. Chem. Acc. 120 (2008) 215-241.

[24] R. A. Motiyenko, L. Margulès, D. Despois, J.-C. Guillemin, Laboratory spectroscopy of methoxymethanol in the millimeterwave range, Phys. Chem. Chem. Phys. 20 (2018) 5509-5516.

[25] J. Demaison, H. Møllendal, J.-C. Guillemin, Equilibrium CAs and CSb bond lengths, J. Mol. Struct. 930 (2009) 21-25.

[26] B. Kirtman, Interactions between ordinary vibrations and hindered internal rotation. I. Rotational energies, J. Chem. Phys. 37 (1962) 2516-2539.

[27] R. Lees, J. Baker, Torsion-vibration-rotation interactions in methanol. I. Millimeter wave spectrum, J. Chem. Phys. 48 (1968) 5299-5318. 
[28] E. Herbst, J. Messer, F. C. De Lucia, P. Helminger, A new analysis and additional measurements of the millimeter and submillimeter spectrum of methanol, J. Mol. Spec. 108 (1984) 42-57.

[29] V. V. Ilyushin, Z. Kisiel, L. Pszczólkowski, H. Mäder, J. T. Hougen, A new torsion-rotation fitting program for molecules with a sixfold barrier: Application to the microwave spectrum of toluene, J. Mol. Spec. 259 (2010) $26-38$.

[30] V. V. Ilyushin, C. P. Endres, F. Lewen, S. Schlemmer, B. J. Drouin, Submillimeter wave spectrum of acetic acid, J. Mol. Spec. 290 (2013) 31-41.

[31] I. Smirnov, E. Alekseev, V. Ilyushin, L. Margulés, R. Motiyenko, B. Drouin, Spectroscopy of the ground, first and second excited torsional states of acetaldehyde from 0.05 to $1.6 \mathrm{THz}$, J. Mol. Spec. 295 (2014) 44-50.

[32] O. Zakharenko, V. V. Ilyushin, F. Lewen, H. S. P. Müller, S. Schlemmer, E. A. Alekseev, M. L. Pogrebnyak, I. A. Armieieva, O. Dorovskaya, L.-H. Xu, R. M. Lees, Rotational spectroscopy of methyl mercaptan $\mathrm{CH}_{3}^{32} \mathrm{SH}_{\text {at }} \mathrm{millimeter}$ and submillimeter wavelengths, A\&A 629 (2019) A73.

[33] R. Suenram, G. Y. Golubiatnikov, I. I. Leonov, J. T. Hougen, J. Ortigoso, I. Kleiner, G. T. Fraser, Reinvestigation of the microwave spectrum of acetamide, J. Mol. Spec. 208 (2001) 188-193.

[34] V. V. Ilyushin, E. Alekseev, S. Dyubko, I. Kleiner, J. T. Hougen, Ground and first excited torsional states of acetamide, J. Mol. Spec. 227 (2004) 115-139.

[35] V. V. Ilyushin, E. Alekseev, S. Dyubko, R. Motiyenko, J. T. Hougen, The rotational spectrum of the ground state of methylamine, J. Mol. Spec. 229 (2005) 170-187.

[36] R. Motiyenko, V. Ilyushin, B. Drouin, S. Yu, L. Margulès, Rotational spectroscopy of methylamine up to 2.6 THz, A\&A 563 (2014) A137.

[37] K. Nakagawa, S. Tsunekawa, T. Kojima, Effective torsion-rotation hamiltonian for methanol-type molecules, J. Mol. Spec. 126 (1987) 329-340.

[38] L.-H. Xu, J. Fisher, R. Lees, H. Shi, J. Hougen, J. Pearson, B. Drouin, G. Blake, R. Braakman, Torsion-rotation global analysis of the first three torsional states $\left(v_{t}=0,1,2\right)$ and terahertz database for methanol, J. Mol. Spec. 251 (2008) 305-313.

[39] H.-W. Kim, M. K. Patel, D. Zeroka, Infrared spectra prediction and potential energy surface studies of methylarsine and methylstibine, Spectroc. Acta A 73 (2009) $730-737$.

[40] D. T. Halfen, D. J. Clouthier, L. M. Ziurys, Millimeter/submillimiter spectroscopy of $\mathrm{PH}_{2} \mathrm{CN}\left(\widetilde{X}^{1} a^{\prime}\right)$ and $\mathrm{CH}_{3} \mathrm{PH}{ }_{2}\left(\widetilde{X}^{1} a^{\prime}\right)$ : probing the complexity of interstellar phosphorous chemistry, ApJ 796 (2014) 36 .

[41] R. J. Gillespie, P. L. Popelier, Chemical bonding and molecular geometry, Oxford University Press,, 2001.

[42] J. Cioslowski, A. Nanayakkara, M. Challacombe, Rapid evaluation of atomic properties with mixed analytical/numerical integration, Chem. Phys. Lett. 203 (1993) 137-142.

[43] J. Cioslowski, P. R. Surján, An observable-based interpretation of electronic wavefunctions: application to "hypervalent" molecules, J. Mol. Struct. 255 (1992) 9-33.

[44] J. Cioslowski, S. T. Mixon, Covalent bond orders in the topological theory of atoms in molecules, J. Am. Chem. Soc. 113 (1991) 4142-4145.

[45] B. B. Stefanov, J. Cioslowski, An efficient approach to calculation of zero-flux atomic surfaces and generation of atomic integration data, J. Comput. Chem. 16 (1995) 1394-1404.

[46] J. Cioslowski, B. B. Stefanov, Variational determination of the zero-flux surfaces of atoms in molecules, Mol. Phys. 84 (1995) 707-716.

[47] J. Cioslowski, A. Nanayakkara, A new robust algorithm for fully automated determination of attractor interaction lines in molecules, Chem. Phys. Lett. 219 (1994) 151-154.

[48] J. Cioslowski, An efficient evaluation of atomic properties using a vectorized numerical integration with dynamic thresholding, Chem. Phys. Lett. 194 (1992) 73-78. 